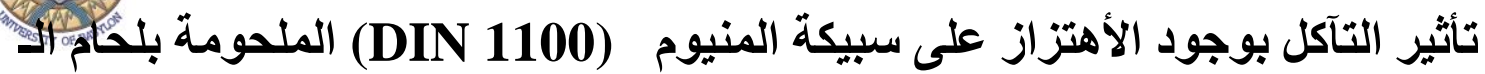
TIG والعاملة في وسط بحري المنيوم
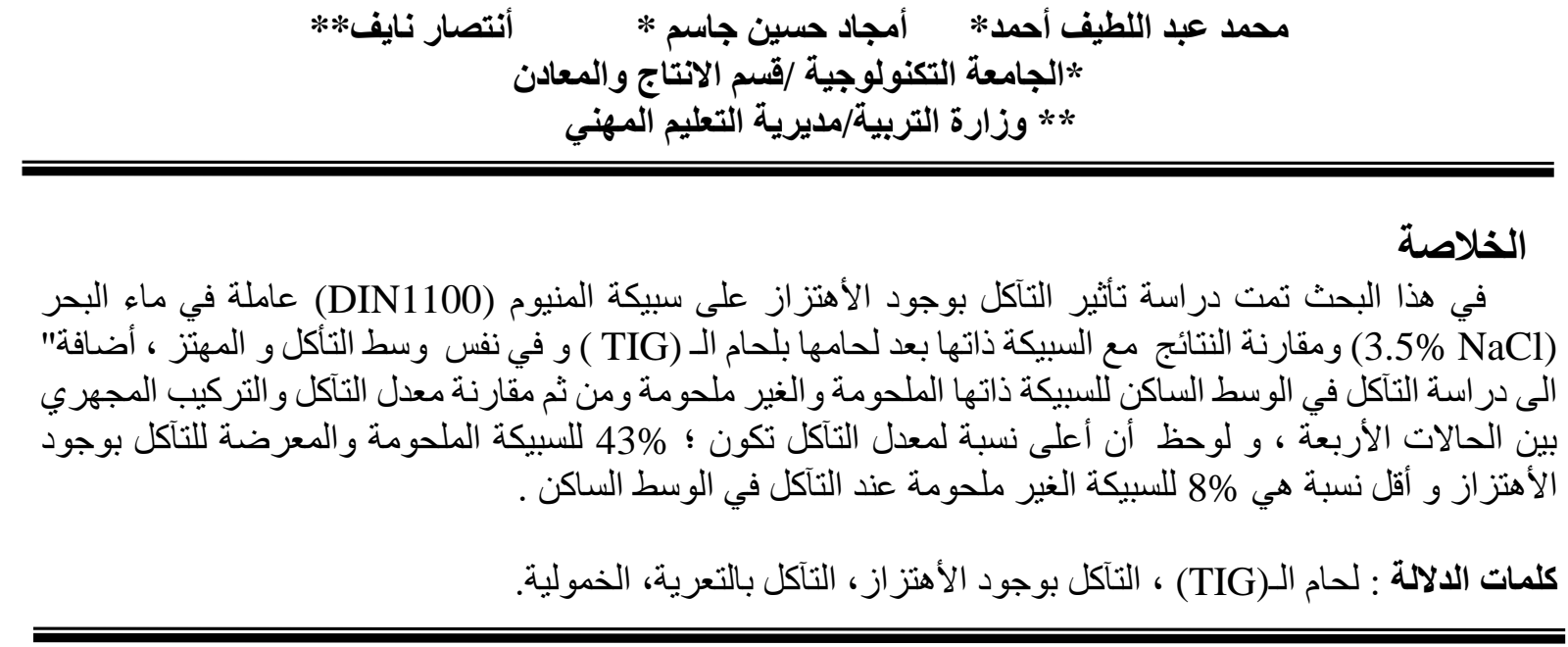

\title{
EFFECT OF VIBRATION-CORROSION ON ALUMINUM ALLOY(DIN1100) THAT WELDED BY TIG TECHNIQUE AND OPERATING IN THE MARINE ENVIRONMENT
}

\author{
"Mohamed Abdu-alalatif Ahmed *Amjad H. Jasim *Entesar Nayyef Farhan \\ en1962farhan@yahoo.com \\ *University of technology / Dept. of Production Engineering and Metallurgy \\ ** Ministry of Education/ Vocational Education
}

\begin{abstract}
In this research studied the effect of corrosion in presence of vibration on aluminum alloy type (DIN1100) operating in sea water $(3.5 \% \mathrm{NaCl})$ and compared these results with the same alloy after it was welded by TIG technique at the same vibrated corrosive medium, addition to study the corrosion in static medium for welded and non- welded alloy, then comparison between the four cases depending on corrosion rates and microstructures it was noted that ; the greatest percentage of corrosion rate was $43 \%$ of welded alloy at vibration-corrosion and least percentage of corrosion rate was $8 \%$ of non-welded alloy at static corrosion.
\end{abstract}

Key world: TIG welding, vibration- corrosion, corrosion erosion, passivity. 


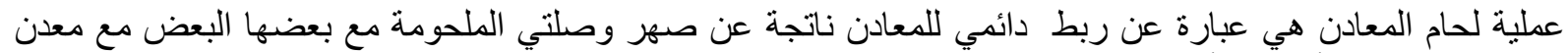

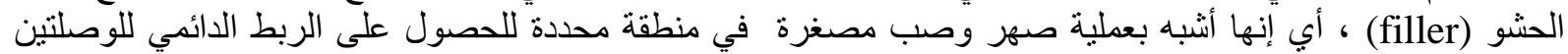

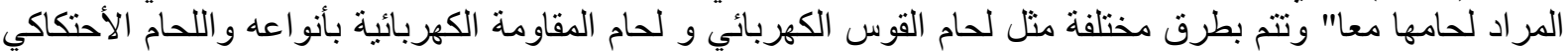

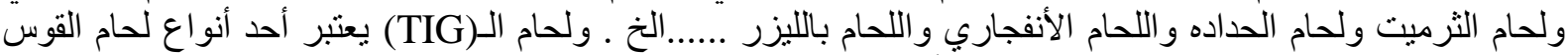

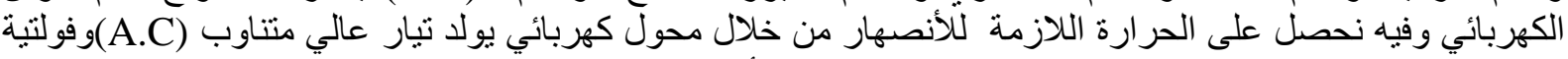

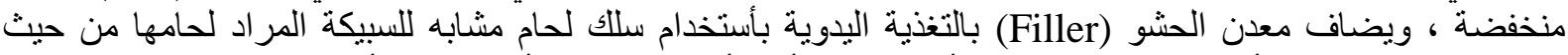

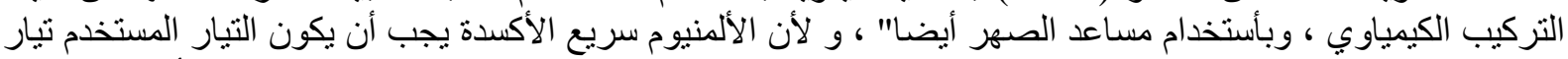
متتاوب (Marks' Standard handbook for Mechanical Engineers] (A.C). سبيكة الألمنيوم(1100)

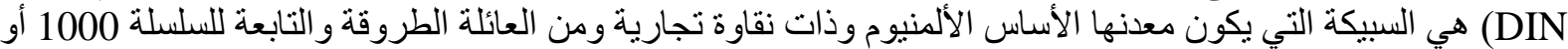
1xxx

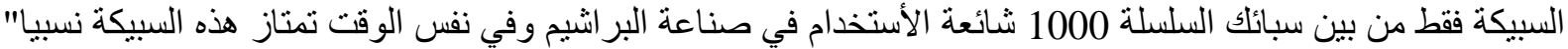

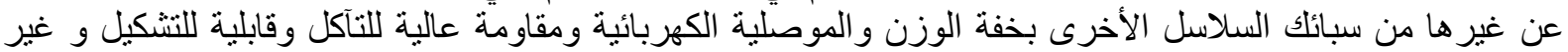

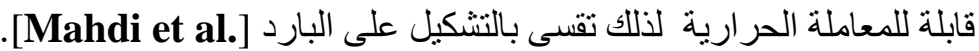

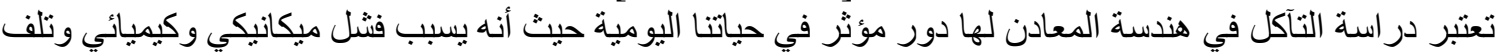

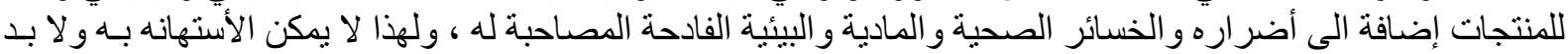

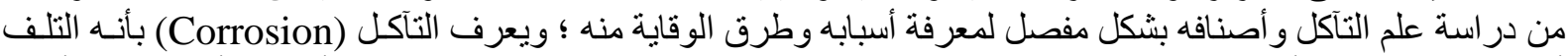

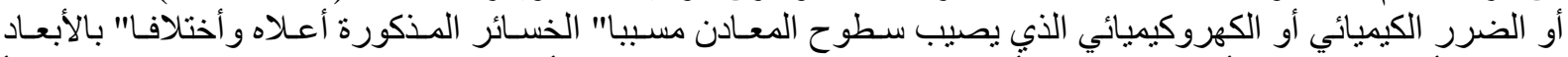

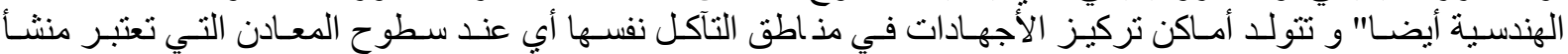

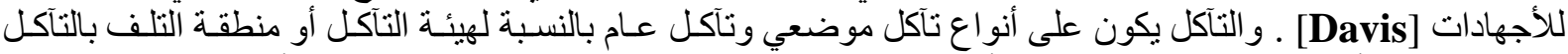

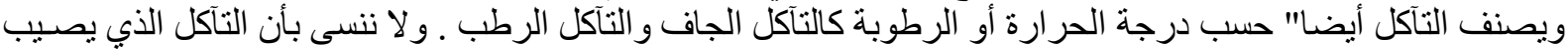

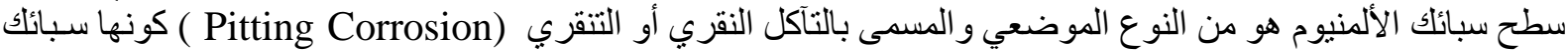

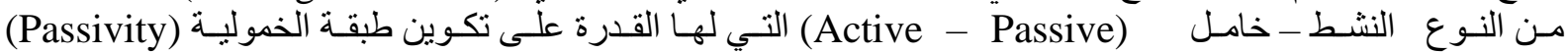

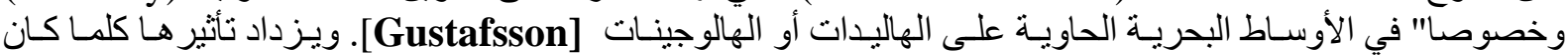

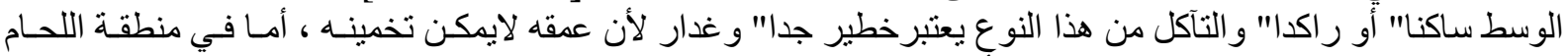

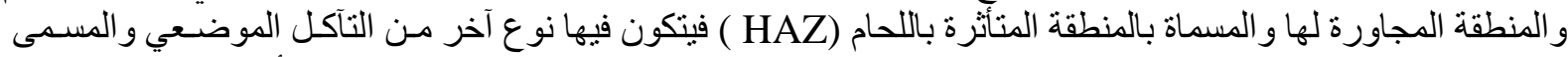

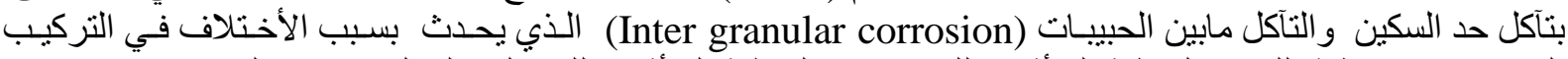

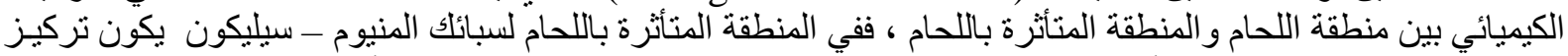

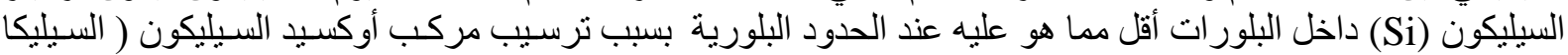

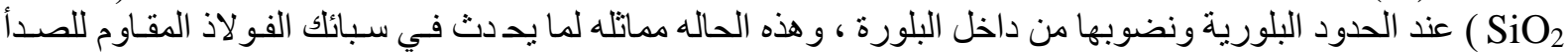

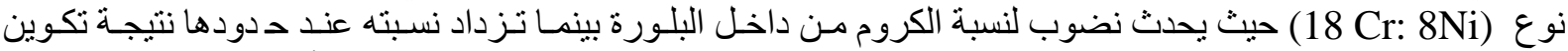

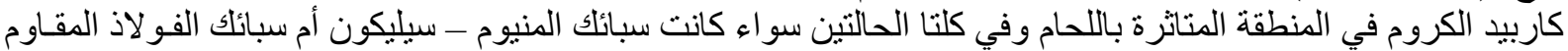

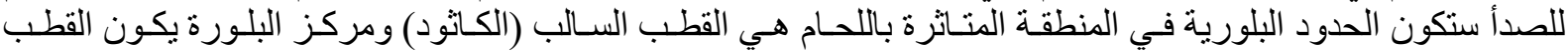

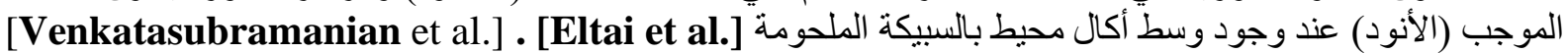

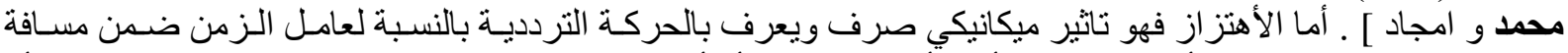

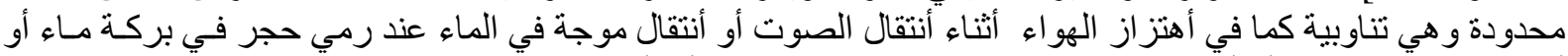

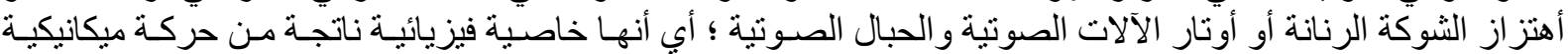

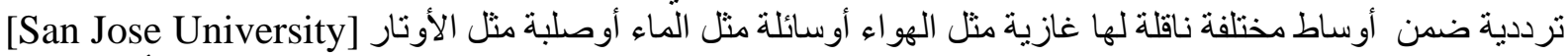
و ت Fundamentals of Electrodynamic Vibration Testing Handbook]

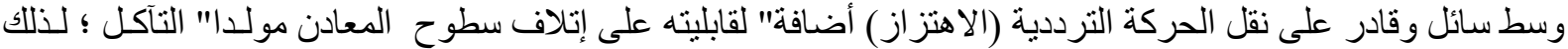

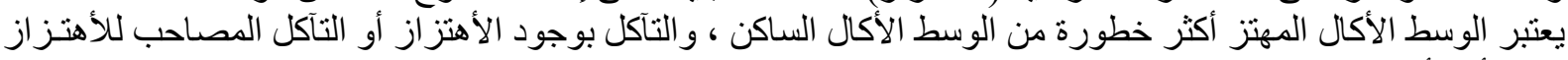

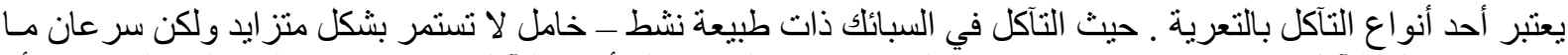

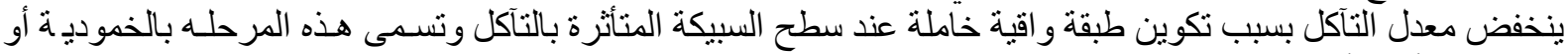

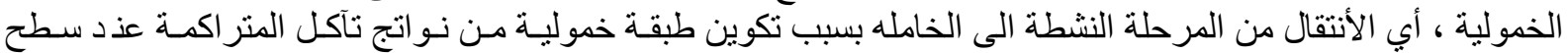

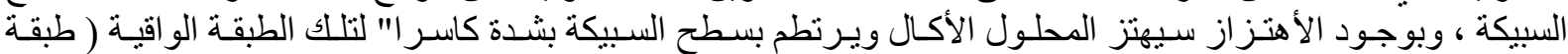
الخمولية) وجار فا" لنو اتج التآكل بعيدا" عن سطح السبيكة تاركة" ور اءولها سطحا" جديدا" نشطا" مستعدا" لأستمر ار التآكل 
وتكرار العمليات ذاتها [ Davis وبهذه الحالة تتولد فجوات عميقة وغير محددة الأشكال خصوصـا" في المنـاطق المختلفـة

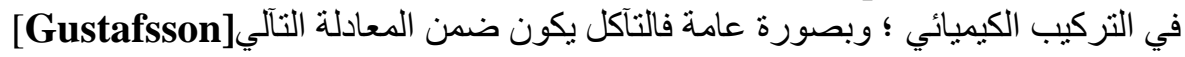

$\mathrm{M}+\mathrm{NaCl} \longrightarrow \mathrm{M}^{+\mathrm{n}} \mathrm{nCl}^{-}+\mathrm{Na}_{2} \downarrow$

يهذف البحث الى دراسة تأثير الأهنز از الميكانيكي المصاحب لعملية التآكل الكيميائي لعينة ذات أساس ألمنيوم ملحومة

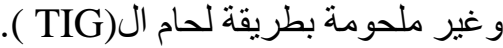

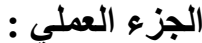
تحضير العينات:

تم تحضير العينات وتهيئتها من سبائك الألمنيوم نوع (DIN 1100) و هي أحدى سبائك الألمنيوم - سيليكون على شكل عينات بأبعاد (5x2x0.5

\section{أيجاد التركيب الكيميائي :}

للتأكد من التركيب الكيميائي للسبيكة بأنها من نوع (DIN 1100) تم إعادة تحليلها كيميائيا" الثركة العامة للتأهيل و الفحص الهندسي وكانت النتائج كما في الجدول رقم (1).

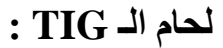

via A.Costa24-40057-Cadriano-Bolonga- نموذج CEBORA تمث عملية اللحام بأستخدام ماكنة نوع

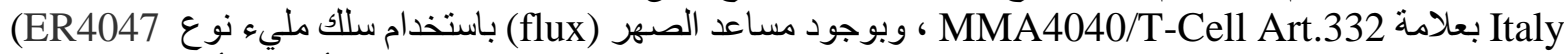

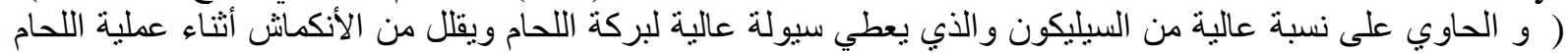

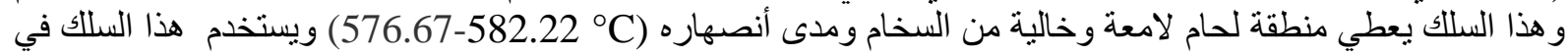
التطبيقات العاملة عند درجات الحرارة المرتفعة ذو نركيب كيميائي نموذجي و المبين في الجدول رانمة رقم (2).

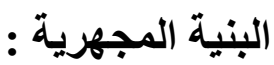
بعد تحضير القطع المعدنية الملحومة والغير الملحومة نم فحص التركيب المجهري لها بأستخدام المجهر الميتالورجي نـــ ( METGT TECH ML 8000 SERIES JAPAN 3003 ) المربوط بالحاسدوب، ولا يتتم تحضير العيذة المعرضة للتآكل لغرض الفحص المجهري تجنبا" لإز الة الأشكال و التضاريس النتاتجة عن التآكل .

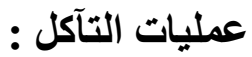

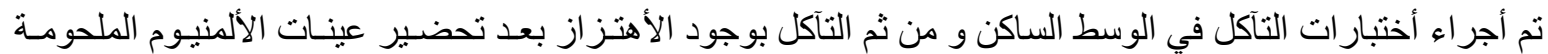

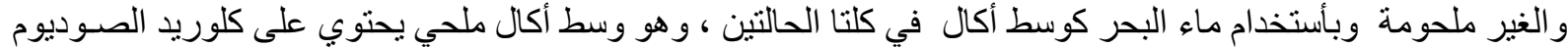

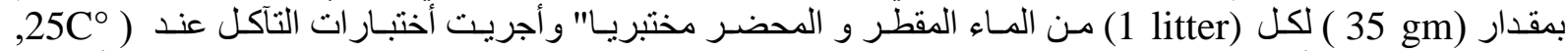
1 atomspher

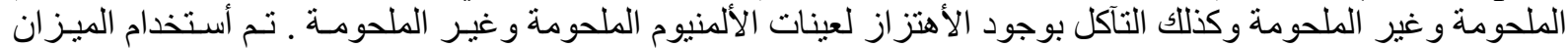

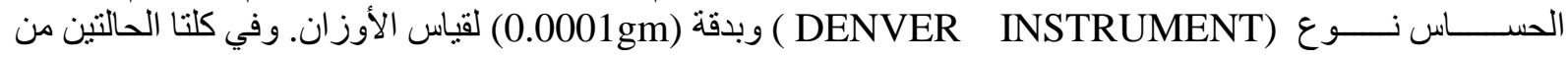

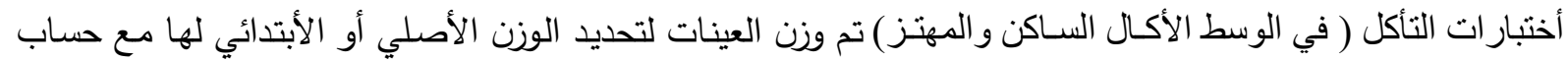

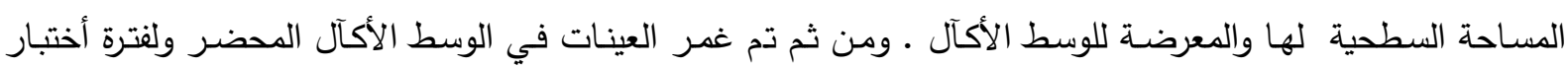
محددة ، وبعد أنتهاء فترة الغمر المحددة لعملية التآكل ؛ أستخرجت العينات من الوسط الأكآل ( الساكن أو المهتز) وتم

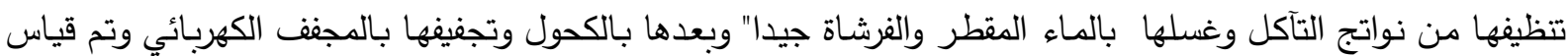
أوزان العينات لتحديد فرق الوزن الحاصل و كالأتي:

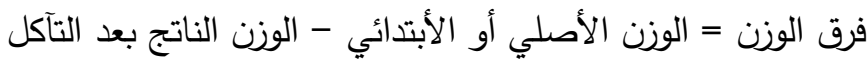

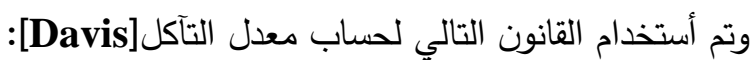

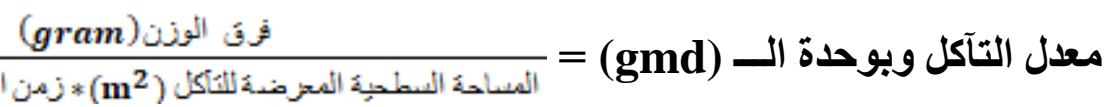




\section{كيفية تحديد معدل التآكل للحالات الأربعة :

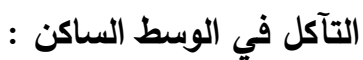

يتم تسجيل الوزن الأبتدائي للعينات ( الملحومة وغير الملحومة ) قبل غمرها في الوسط الأكال الساكن أي عندما يكون

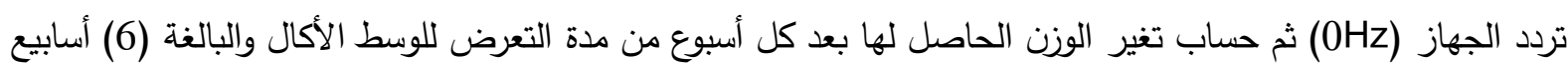

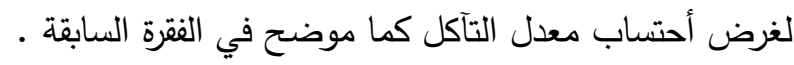

التآكل بوجود الأهتزاز (الوسط مهتز) : في حالة التآكل بوجود الأهتزاز يستخدم نفس الوسط الأكال في حالة التآكل في الوسط الساكن ، ولكن موضوع داخل حجرة

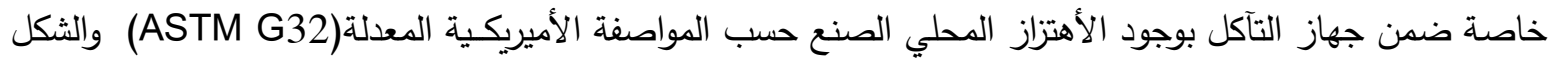

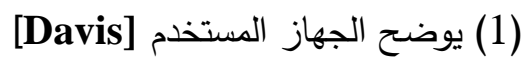
وهنالك خطوات أساسية يجب مراعاتها في التجارب المختبرية الخاصة بالتآكل بوجود الأهتزاز ليتم أعتمادها في دراسة تاثير

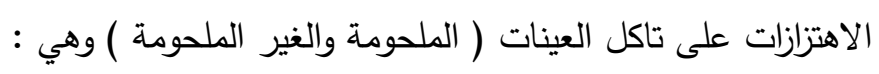

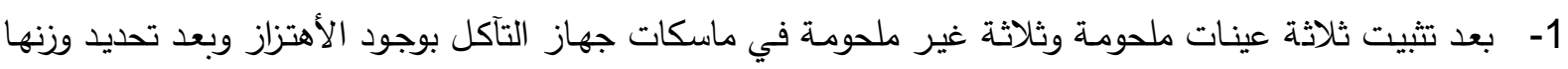

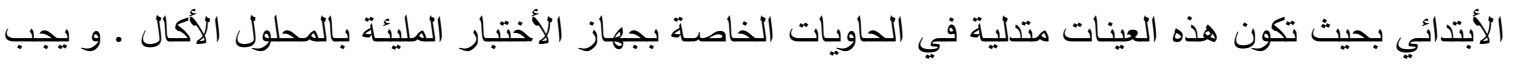

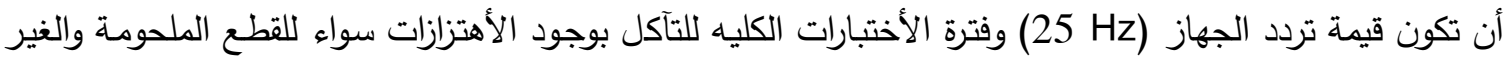

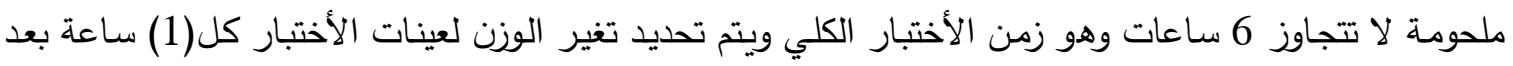

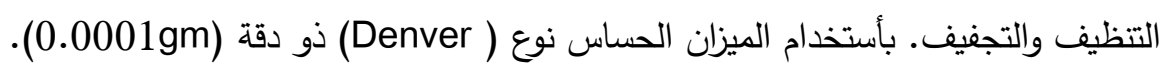

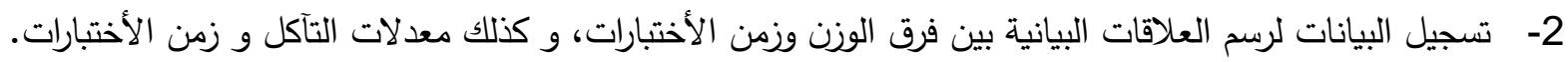

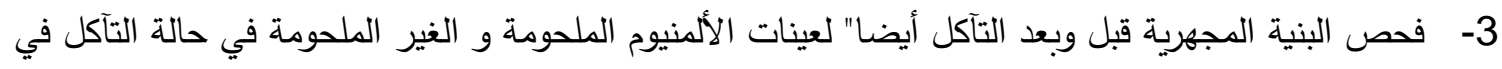
الوسط الساكن أو الوسط المهتز للمقارنة بين الحالات الأربعة من حيث البنية المجهرية.

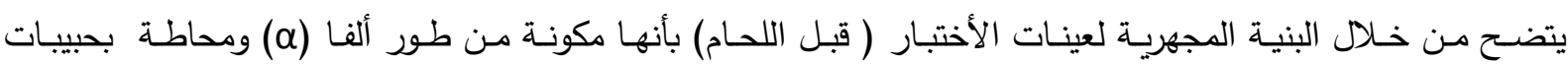

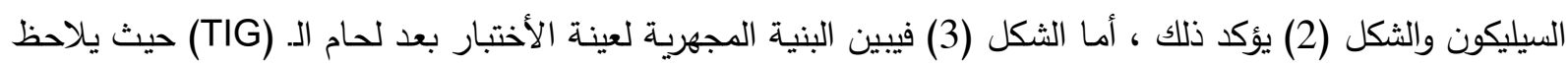

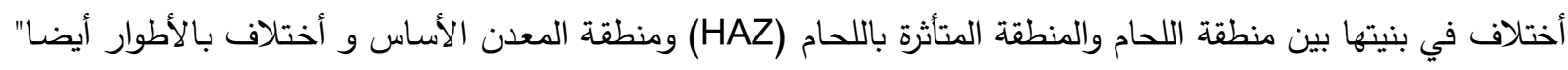

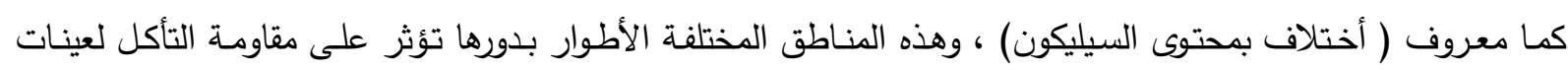

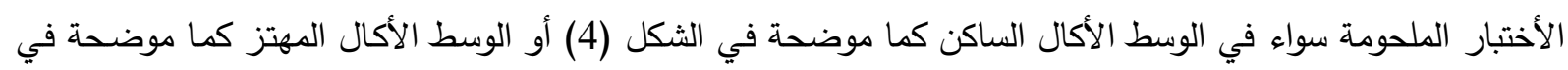

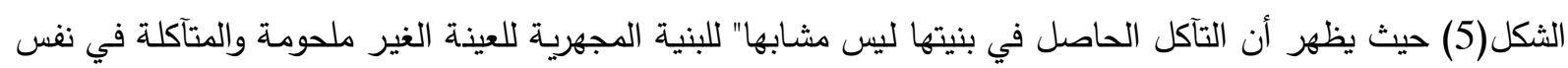

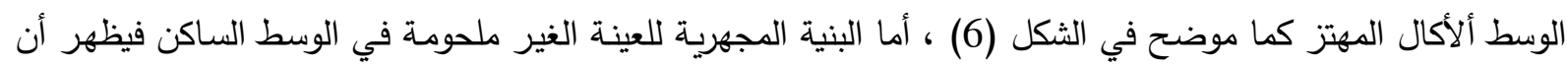

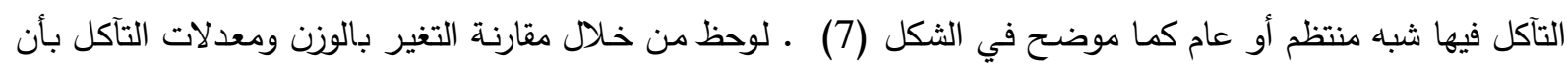


تأثير التآكل بوجود الأهتزاز يكون أكثر شدة من التآكل في الوسط الأكال الساكن نتيجة الأهتزاز المتزامن مع التآكل و

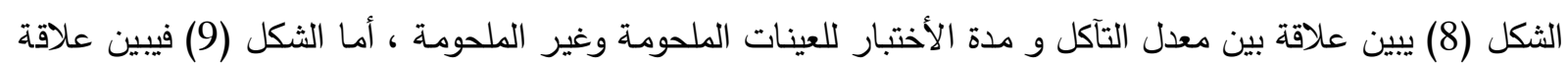
معدل التآكل مع مدة الأختبار للعينات الملحومة وغير الملحومة في الوسط الأكال المهتز وهذا يطابق التغيرات في البنية المجهرية الموضحة أعلاه. وللمقارنة بين حالات التآكل الأربعة أعلاه فأن الثكل (10) يبين المقارنة بين النسبة المئوية لمعدلات التآكل و بين حالات التآكل الأربعة.

\section{الأستنتاجات}

يلاحظ من المخططات البيانية و البنية المجهرية للحالات المختلفة أعلاه بأن معدل التآكل بوجود الأهتزاز يكون ذو تأثير

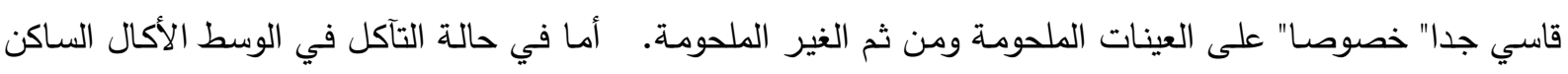

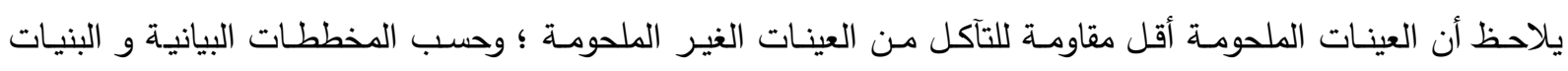
المجهرية ـ والثكل (10) يبين المقارنة بين النسبة المئوية لحالات التآكل الأربعة وهو دليل واضئح على صلى صحة النتائج .

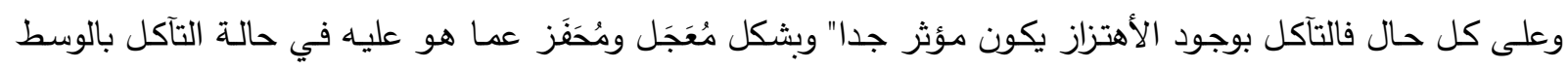

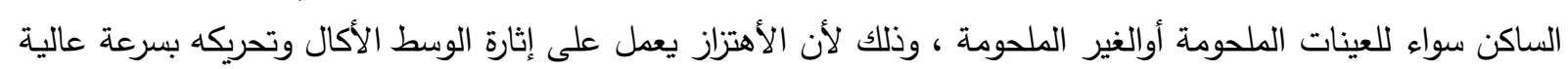

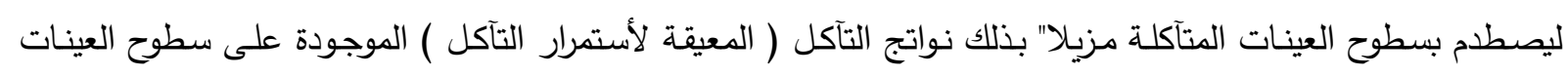

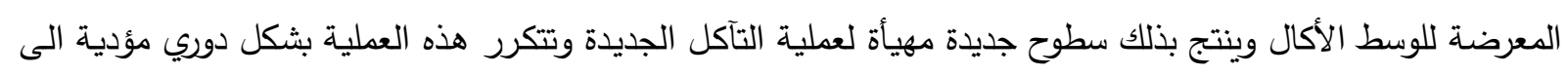
أنهيار شديد للعينات المتآكلة في حالة أستمرار الأهتزاز المصاحب للتآكل. أما بالنسبة لأختلاف شدة التآكل بين العينات التهات

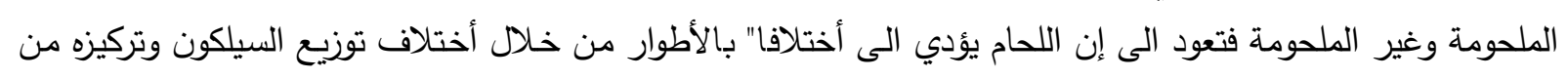

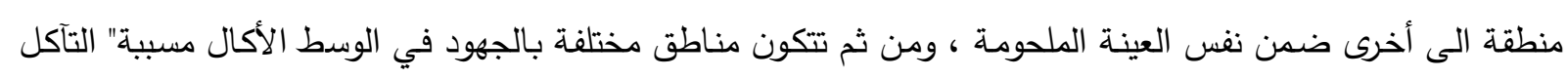

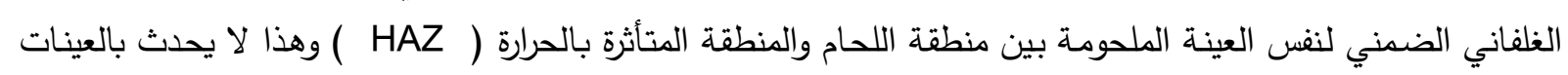

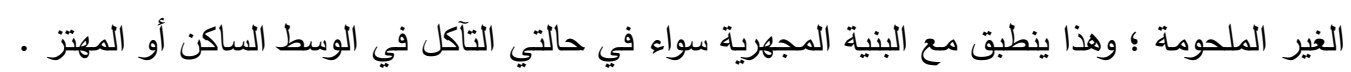

جدول رقم (1): التركيب الكيميائي لسبيكة الألمنيوم المستخدمة.

\begin{tabular}{|c|c|c|c|c|c|c|c|c|c|c|}
\hline عنصر & $\mathrm{Si}$ & $\mathrm{Fe}$ & $\mathrm{Cr}$ & $\mathrm{Cu}$ & $\mathrm{Mn}$ & $\mathrm{Mg}$ & $\mathrm{Ti}$ & $\mathrm{Zn}$ & $\mathrm{Be}$ & $\mathrm{Al}$ \\
\hline نسبيته & 0.3 & 0.5 & 0.016 & 0.1 & 0.03 & 0.05 & 0.001 & 0.09 & 0.0002 & Rem. \\
\hline
\end{tabular}

جدول رقم (2) يبين التركيب الكيميائي النموذجي لسلك اللحام نوع (ER4047) [10]

\begin{tabular}{|c|c|c|c|c|c|c|c|c|}
\hline المعدن & $\% \mathrm{Si}$ & $\% \mathrm{Fe}$ & $\% \mathrm{Cu}$ & $\% \mathrm{Mn}$ & $\% \mathrm{Mg}$ & $\% \mathrm{Zn}$ & $\% \mathrm{Be}$ & $\% \mathrm{Al}$ \\
\hline الفقدار & 12.5 & 0.5 & 0.15 & 0.065 & 0.05 & 0.08 & 0.0001 & Rem. \\
\hline القياسي & $\begin{array}{l}11.0- \\
13.0\end{array}$ & $\begin{array}{c}0.8 \\
\max \end{array}$ & $\begin{array}{c}0.3 \\
\max \end{array}$ & $\begin{array}{l}0.15 \\
\max \end{array}$ & $\begin{array}{c}0.1 \\
\max \end{array}$ & $\begin{array}{c}0.2 \\
\max \end{array}$ & $\begin{array}{c}0.0003 \\
\max \end{array}$ & Rem. \\
\hline
\end{tabular}




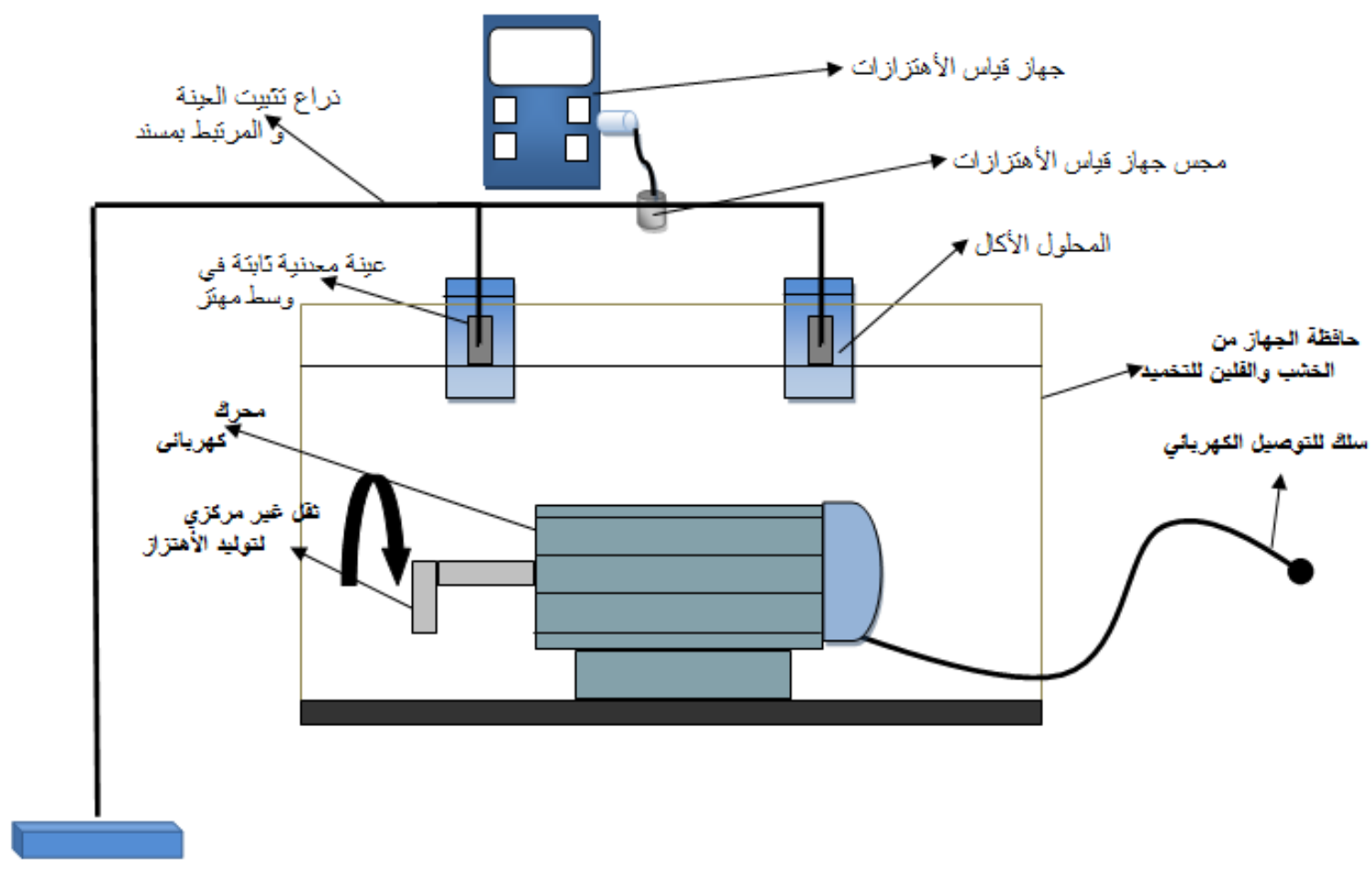

شكل(1) يوضح جهاز التآكل بوجود الأهتزاز •

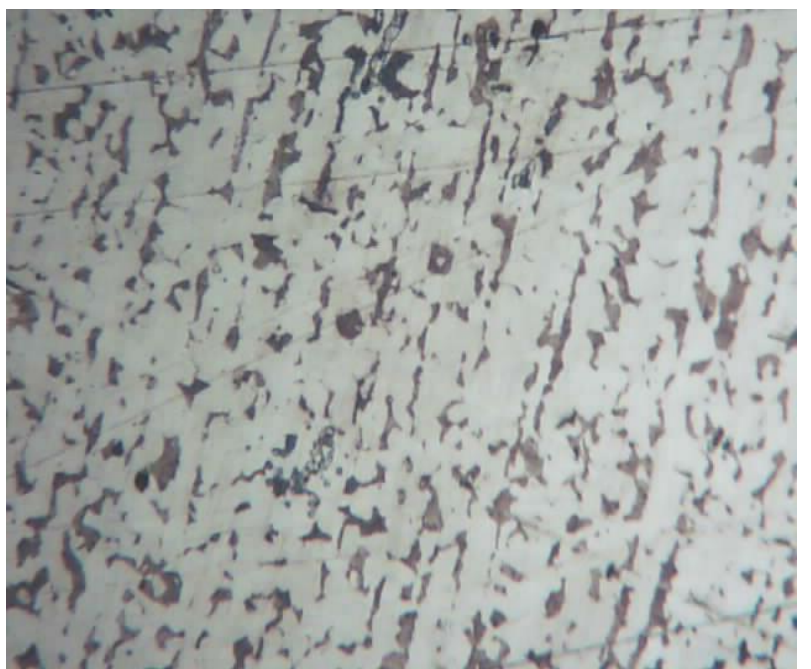

شكل(2) البنية المجهرية لعينة الألمنيوم قبل اللحام و تأثير التاكل.

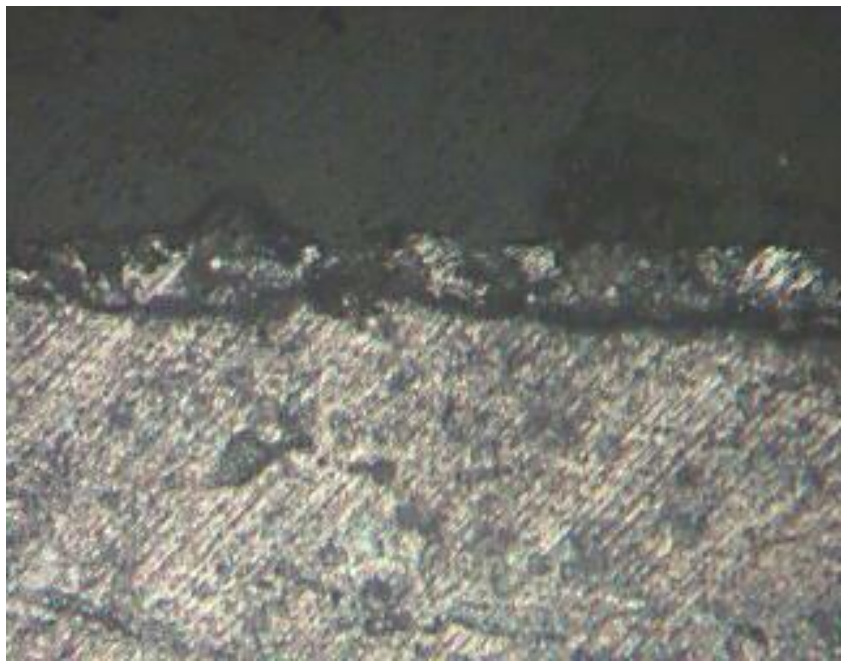

شكل(3) البنية المجهرية لعينة الأختبار بعد لحام الـ .(TIG) 
MohamedA.etal. The Iraqi Journal For Mechanical And Material Engineering, Vol.20, No.1March. 2020
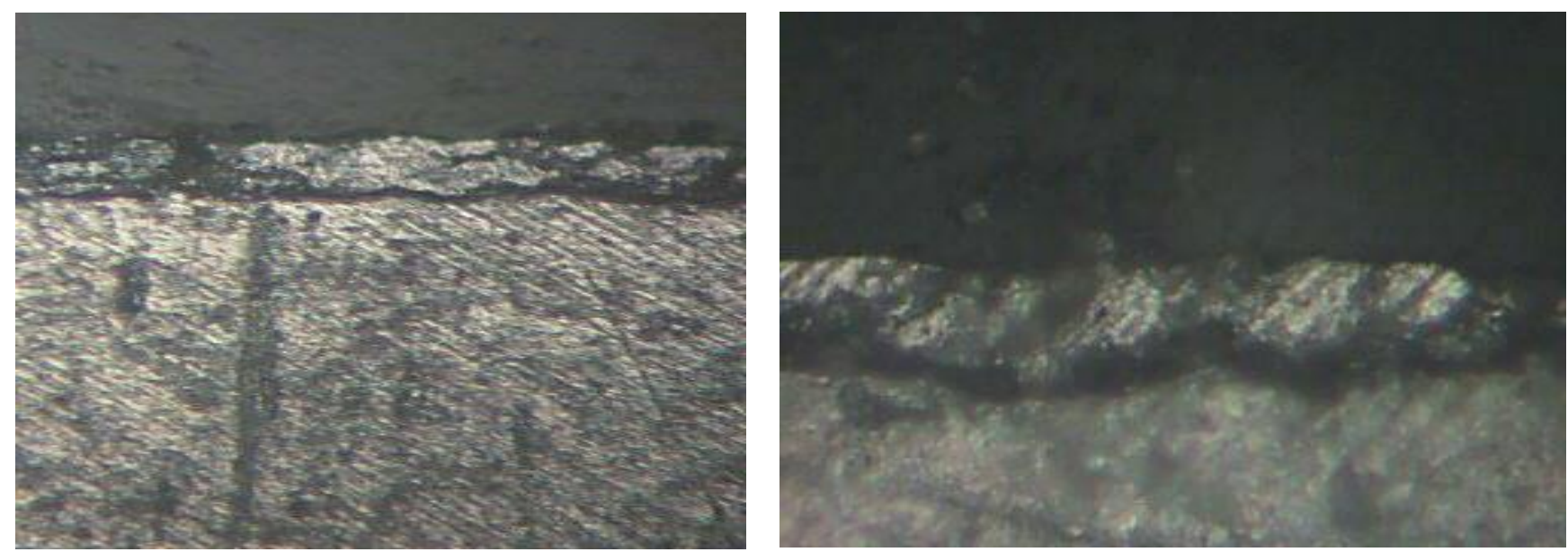

شكل (5) البنية المجهرية لعينة الأختبار الملحومة بعد شكل (4) البنية المجهرية لعينة الأختبار الملحومة بعد التآكل في الوسط الساكن

التآكل في الوسط المهتز •

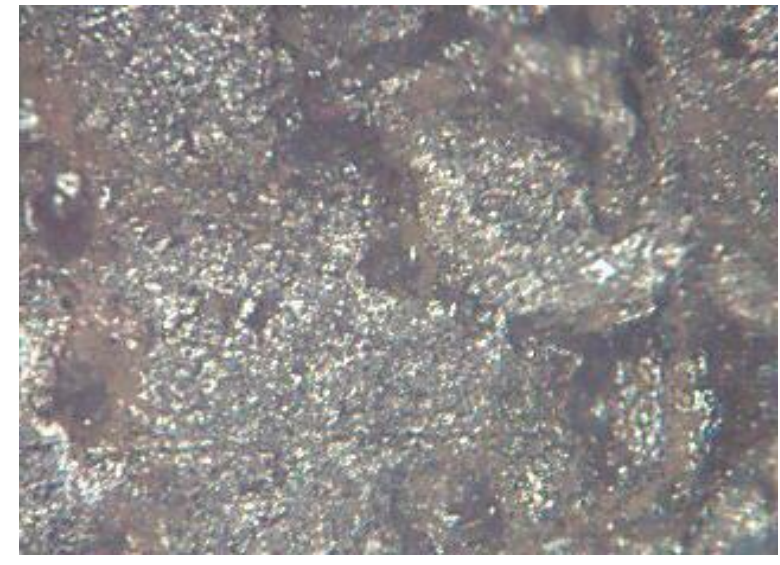

شكل (6) البنية المجهرية لعينة الأختبار الغيرملحومة

بعد التآكل في الوسط المهتز .

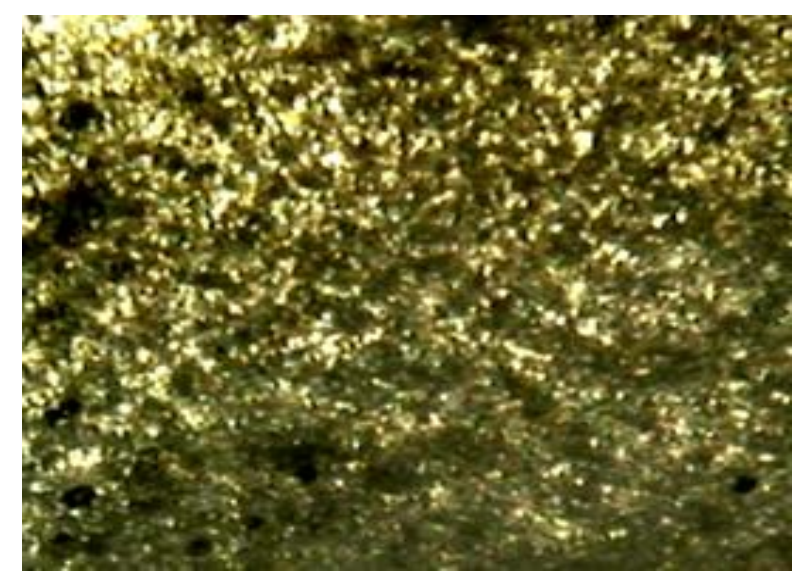

شكل (7) يوضح البنية المجهرية لعينة الأختبار الغيرملحومة بعد التآكل في الوسط الساكن. 


\section{مقارنة التآكل في الوسط الساكن قبل وبعد لحام الـ} TIG
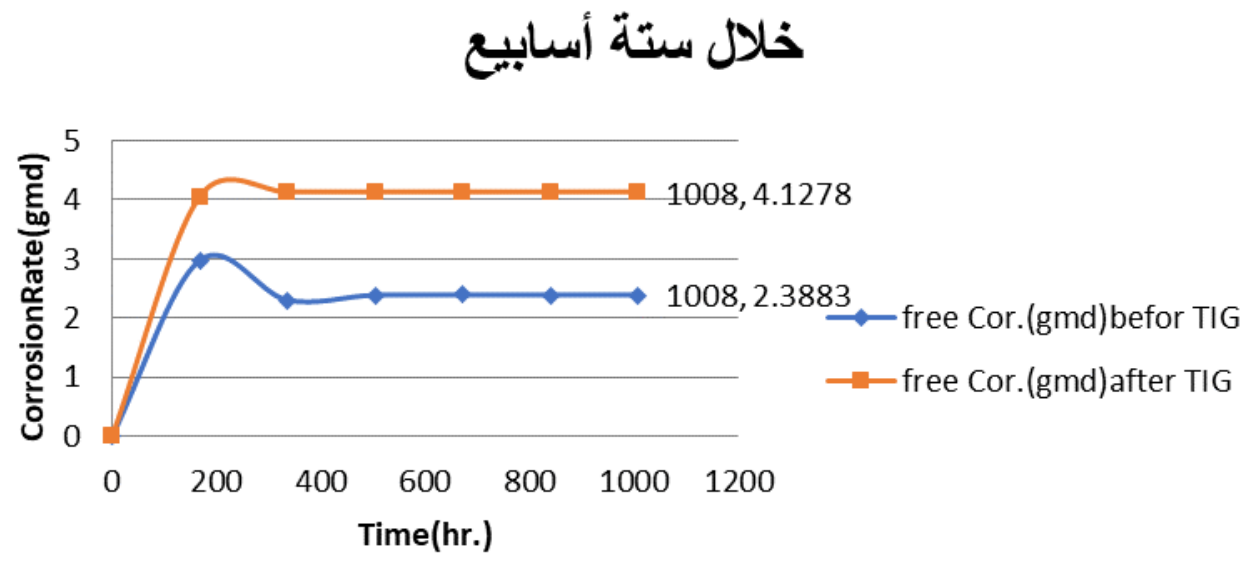

شكل (8) يوضح مقارنة التآكل الحاصل في الوسط الساكن قبل وبعد عملية اللحام بال (TIG ) مقارنة التآكل بوجود الأهتزاز قبل وبعد لحام الـTIG خلال ستة ساعات

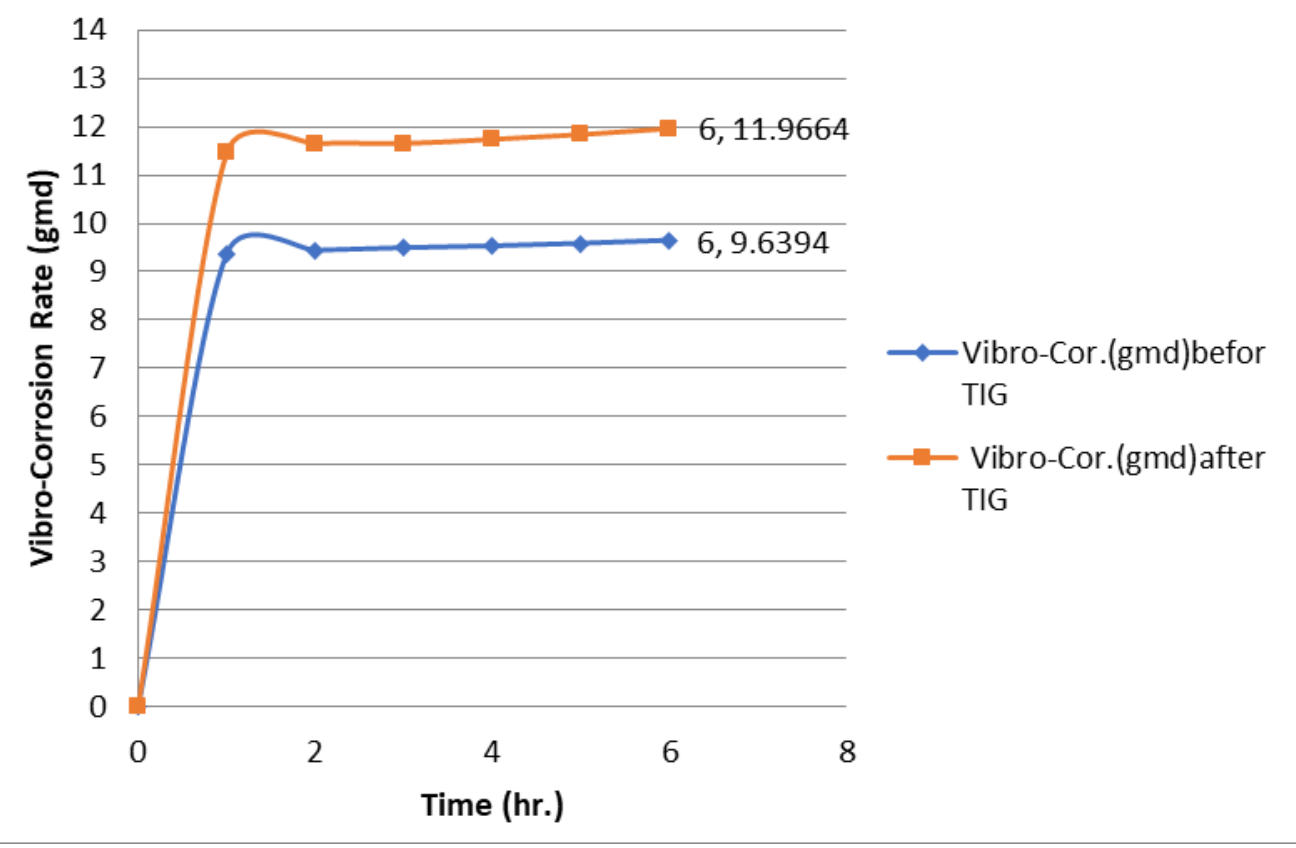

شكل (9) يبين مقارنة التآكل بوجود الأهتزاز قبل وبعد عملية اللحام بال (TIG ). 


\section{المقارنة بين النسب المئوية لحالات التآكل الأربعة}

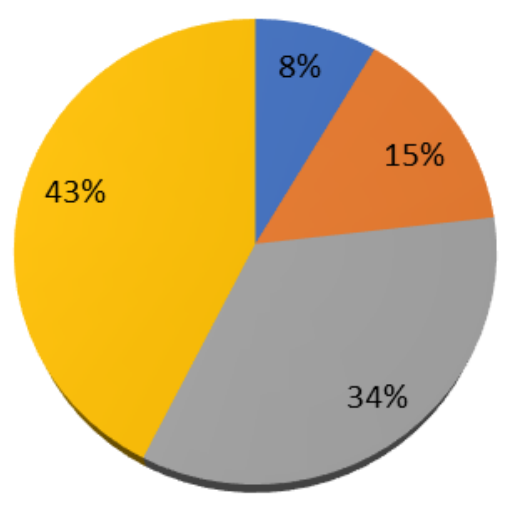

- Cor.(gmd)befor TIG

- Cor.(gmd)after TIG

- Vibro-Cor.(gmd)befor TIG

- Vibro-Cor.(gmd)after TIG

\section{شكل (10) المقارنة بين النسب المئوية لحالات التآكل الأربعة}

\section{REFERENCES}

Elsadig. Eltai , E. Mahdi, Akram Alfantazi, "The Effects of Gas Tungsten Arch Welding on the Corrosion and Mechanical Properties of AA 6061 T6", Qatar University, Department of Mechanical and Industrial Engineering, P.O. Box 2713, Doha, Qatar, Int. J. Electrochem. Sci., 8 (2013) 7004 - 7015.

Farouk M. Mahdi, Abdul Mun'em A. Karim, Eman G. Gi'baz "Study of pitting corrosion behavior for Aluminum Alloy 1100in 3\% NaCl solution" gDiyala Journal of Engineering Sciences, University of Diyala, Vol.2 ,No.1 ,June 2009.

J.R. Davis, "Corrosion of aluminum and aluminum alloys" (\#06787G), Copyright 1999 ASM International @ All Right reserved, www..asminternational.org.

Marks' Standard handbook for Mechanical Engineers, $8^{\text {th }}$ Ed., Mc Graw Hill, pp.6-50to 6-58.

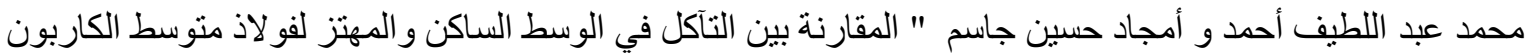

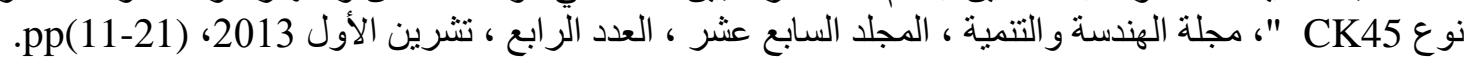

Sofia Gustafsson, "Corrosion properties of aluminum Alloys and surface treated alloys in tap water”, UPTEC K 11028, Examensarbete30hp, Uppsala university, June 2011.

Venkatasubramanian G., Sheik Mideen A and Aboy K. Jha, "Effect of pH on the Corrosion Behavior of Aluminium Alloy Welded Plate in Chloride Solutions", Department of Chemistry, Sathyabama University, Jeppiaar Nagar, Chennai-600119, INDIA, Research Journal of Chemical Sciences, ISSN 2231-606X Vol. 3(6), 74-80, June (2013).

Fundamentals of Electrodynamic Vibration Testing Handbook, Written and published by Thermotron Industries, Holland, MI Copyright 2006. 
San Jose State University, Department of Mechanical and Aerospace , Engineering,ME 120 Experimental Methods, Vibration Measurement, BJ , Furman 22NOV05, BJ Furman SJSU MAE.

MAXAL Guide for Aluminum Welding “ The Welder's Choice for Quality Aluminum Weld Wire", maxal.com, Maxal International.Inc., June 2011. 